\title{
La competitividad de América Latina en el comercio de servicios basados en el conocimiento
}

\author{
Andrés López, Andrés Niembro y Daniela Ramos
}

RESUMEN

En este trabajo se presenta un análisis dinámico de la competitividad de América Latina en los "servicios intensivos en conocimiento" (SIC), utilizando una metodología del estilo TradeCAN — desarrollada por la Comisión Económica para América Latina y el Caribe (CEPAL) - para evaluar la competitividad de un país mediante sus exportaciones a los mercados de mayor crecimiento, pero aplicada principalmente al comercio de bienes. Los resultados sugieren que si bien varios países latinoamericanos avanzaron en los segmentos de los SIC y muestran ventajas comparativas positivas, existe aún un bajo porcentaje de "estrellas nacientes" (sectores dinámicos donde se gana participación de mercado) y un relativamente alto nivel de "oportunidades perdidas" (sectores dinámicos donde se pierde peso), lo que se corresponde con las debilidades competitivas de la región y plantea la necesidad de políticas para potenciar los factores aventajados y remover los obstáculos que traban una mejor inserción en los mercados de los SIC.

PALABRAS CLAVE

CLASIFICACIÓN JEL

AUTORES
Competitividad, comercio de servicios, economía basada en el conocimiento, comercio internacional, exportaciones, estadísticas comerciales, América Latina

E01, F14, L80

Andrés López, investigador del Centro de Investigaciones para la Transformación (cenit/untref), Argentina.anlopez@fund-cenit.org.ar

Andrés Niembro, investigador del Centro Interdisciplinario de Estudios sobre Territorio, Economía y Sociedad (cietes), Universidad Nacional de Río Negro (Sede Andina), Argentina. Becario Doctoral del ConicET, Argentina. aniembro@unrn.edu.ar

Daniela Ramos, investigadora del Centro de Investigaciones para la Transformación (cenit/untref), Argentina.danielaramos@fund-cenit.org.ar 


\section{I}

\section{Introducción}

Los servicios son desde hace tiempo un sector dominante en la economía global, ya que representan la mayor porción del producto interno bruto (PIB) y el empleo tanto en las economías desarrolladas como en buena parte del mundo en desarrollo.

Hasta hace poco el sector de servicios era considerado como sinónimo de baja productividad, poca innovación y - salvo en algunas actividades como transporte y turismo- de escasa o nula posibilidad de efectuar transacciones. Sin embargo, en las últimas décadas se asiste a enormes transformaciones, que han dado lugar a un nuevo paradigma en la producción y el comercio de servicios y que le otorgan a estas actividades un papel completamente diferente del que tenían en el pasado dentro de las economías nacionales.

Dos fenómenos clave se destacan en este sentido. El primero es la creciente relevancia de un conjunto de actividades denominadas "servicios intensivos en conocimiento" (SIC) (knowledge intensive services). En esta categoría se agrupa a segmentos tan diversos como servicios contables y legales, audiovisuales, diseño, publicidad, programas computacionales (software) y servicios informáticos, investigación y desarrollo (I+D), salud y educación ${ }^{1}$. El factor común a todos ellos es el hecho de que emplean intensivamente capital humano de alto nivel de calificación y son usuarios y productores de información y conocimiento para prestar servicios a sus clientes.

El segundo fenómeno importante es que mientras varios de estos servicios eran prestados sobre todo fronteras adentro hasta no hace muchos años, ahora pueden ser exportados. En las estadísticas disponibles no se capta totalmente este proceso, si se consideran, entre otros factores, la naturaleza intangible del comercio de servicios y las consecuentes dificultades de registro. Sin embargo, aun sabiendo que las cifras conocidas son, en todo caso, un piso del verdadero valor del comercio de servicios, al presente se estima que alrededor del $20 \%$ de

\footnotetext{
${ }^{1}$ Dado que el interés del presente trabajo se centra en la competitividad, los SIC se identificarán a partir de los datos de comercio disponibles sobre los siguientes rubros: servicios empresariales y profesionales, programas computacionales (software) y servicios informáticos, y servicios audiovisuales, culturales y personales, lo que si bien redunda en una cobertura imperfecta del concepto de sIC, es una aproximación suficientemente buena para los fines del análisis en este trabajo.
}

las exportaciones mundiales totales (bienes más servicios) corresponden a este último sector. Incluso más interesante es que estimaciones efectuadas recientemente sugieren que si se mide el comercio en términos de valor agregado (sobre la base del uso de matrices insumo-producto), esa participación bordea el 50\% y supera a la del comercio de manufacturas (Escaith, 2008).

El avance del comercio de servicios ha sido liderado por los SIC (véase el gráfico 1). Actualmente, las exportaciones globales de dichos servicios llegan a cerca de 1,6 billones de dólares (datos de 2012 de la Conferencia de las Naciones Unidas sobre Comercio y Desarrollo UNCTAD), cifra 3,7 veces superior a la del año $2000^{2}$. El comercio de este tipo de servicios en 2012 superó, por ejemplo, al de alimentos (incluidos materias primas y procesados), minerales, acero, textiles y confecciones, electrónicos o automóviles, solo por citar algunas industrias conocidas y relevantes a escala global.

El dinamismo del comercio de los sic ha venido de la mano del despliegue de las tecnologías de la información y las comunicaciones (TIC), que han abaratado y acelerado notablemente la generación, almacenaje, procesamiento y transmisión de la información, e incluso han permitido digitalizar ciertos bienes y convertirlos en intangibles. Esas mismas TIC han facilitado que ciertos servicios que antes requerían del contacto físico directo entre prestador y usuario ahora puedan ser provistos de manera remota.

A su vez, las TIC son la base infraestructural de los procesos de fragmentación de la producción a escala mundial, que han dado lugar a la formación de cadenas globales de valor (CGV). Dentro de esas cadenas, los procesos de producción se componen de "porciones" de valor agregado que son generadas en distintas localizaciones. Así, lo relevante ya no es más qué se produce en un determinado país o región, sino qué tareas se desarrollan allí y cuál es el valor agregado que generan (Baldwin, 2011). Las empresas transnacionales juegan un papel central como organizadoras de la mayor parte de las CGV, en la medida en que procuran "deslocalizar" su provisión de bienes y servicios allí donde pueden encontrar costos competitivos y acceso a recursos calificados.

\footnotetext{
${ }^{2}$ Si se suman los servicios financieros y seguros, las comunicaciones y regalías y las licencias, la cifra trepa a 2,35 billones de dólares en 2012.
} 
GRÁFICO 1

Composición del comercio internacional de servicios, 2000-2012

(En porcentajes del total)

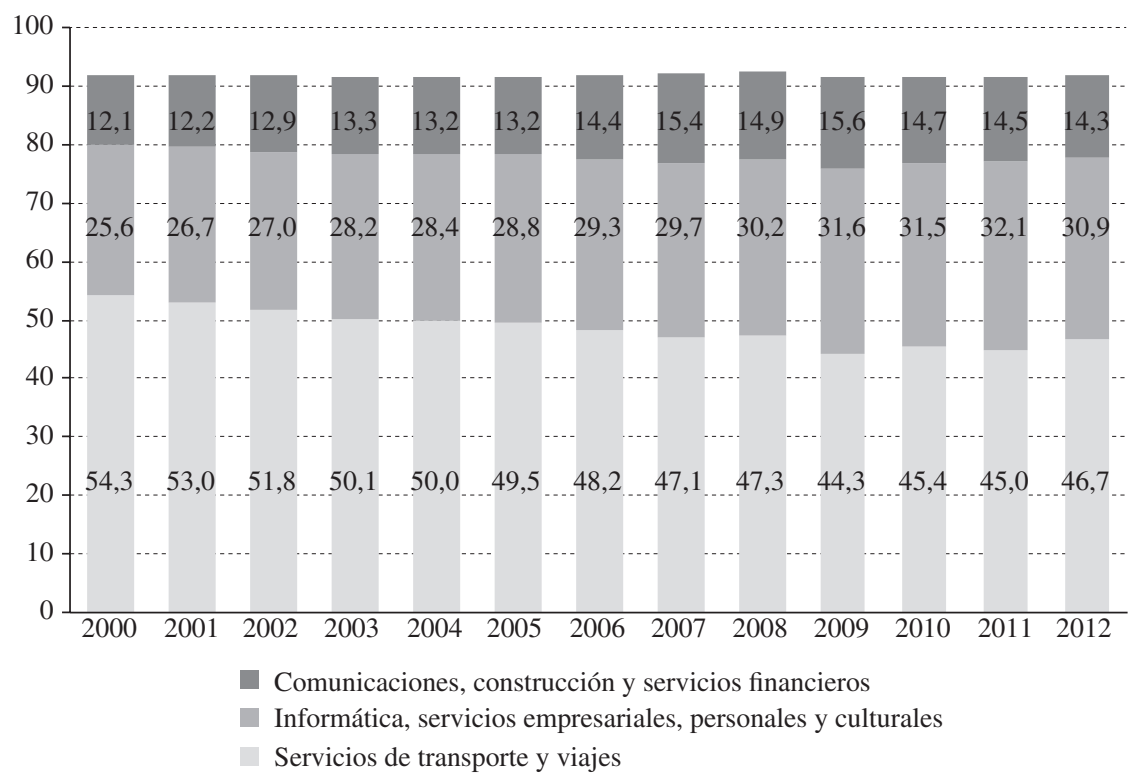

Fuente: elaboración propia sobre la base de datos de la Conferencia de las Naciones Unidas sobre Comercio y Desarrollo (UNCTAD).

Nota: las categorías del gráfico no suman $100 \%$, pues se excluyeron los valores de "regalías y licencias" y "servicios de gobierno".

Si se pone el foco en los sic, China y la India, sobre la base de sus vastas reservas de fuerza de trabajo con educación universitaria, han alcanzado un claro liderazgo en el mercado global. Según datos de la UNCTAD, ambos países exportan más de 90 mil millones de dólares de SIC. Pero existe un gran número de países en desarrollo que han logrado insertarse en estos mercados, incluidos otros asiáticos como Malasia, Filipinas o Tailandia y varios de Europa oriental (Polonia, República Checa, Hungría), además de casos emblemáticos en el mundo desarrollado, como el de Irlanda.

En este contexto, América Latina también ha comenzado a participar activamente de esta nueva dinámica del comercio global y varias naciones de la región muestran un dinamismo apreciable en sus exportaciones de SIC (especialmente en los casos de la Argentina, el Brasil, Colombia, Costa Rica, Chile y el Uruguay ${ }^{3}$ ).

Surge entonces el interés por ver, más allá de las cifras de comercio, cuán competitiva es la región en los mercados de los sIC. Como es sabido, la noción de "competitividad internacional" involucra una gran

\footnotetext{
${ }^{3}$ Un hecho que vale la pena destacar, puesto que incide en el desarrollo de este trabajo, es que México no reporta estadísticas para exportaciones de los SIC, salvo en el caso de audiovisuales, lo que explica la ausencia de dicho país en varios de los cuadros aquí presentados.
}

cantidad de factores y, por ende, ha dado origen a una amplia variedad de concepciones y enfoques. Salvando las dificultades existentes para consensuar una única definición, se puede sugerir que, en términos generales, la competitividad se refiere al desempeño (relativo) de una firma, sector o país en la economía internacional. A partir de esta idea, se han desarrollado diversos indicadores con el fin de comparar el rendimiento relativo de las naciones/empresas en los mercados mundiales.

Aunque es cada vez más frecuente enmarcar a la competitividad internacional como parte de un proceso complejo y dinámico (estructural, sistémico, u otros), en el que interactúan factores micro, meso y macroeconómicos, la mayoría de los análisis disponibles sobre la competitividad de distintos países en el comercio internacional de servicios suelen limitarse a la utilización del indicador de "ventajas comparativas reveladas" (véanse, por ejemplo, Langhammer, 2004; Díaz de la Guardia, Molero y Valadez, 2005; Bobirca y Miclaus, 2007; Seyoum, 2007; Zhang, Wei y Miao, 2010; Jiang, 2011). Este tipo de enfoque metodológico se reduce a un cálculo ex post (para un determinado momento) del grado de especialización de la economía en el comercio mundial y se asienta en una concepción fundamentalmente estática de las ventajas comparativas (Ferraz, Kupfer y Haguenauer, 1996). Como señalan Chudnovsky y Porta 
(1990, págs. 13-15), esta noción teórica (tradicional) de ventajas comparativas estáticas no es demasiado útil a la hora de analizar posibles áreas de intervención dirigidas a crear ventajas dinámicas ${ }^{4}$ y extraer mayores beneficios de la participación en el comercio internacional.

En este marco, el propósito del presente artículo es aportar una forma alternativa de análisis de la competitividad internacional de América Latina en el sector de los SIC, desde una óptica que, si bien trabaja sobre resultados ex post, apunta a conocer en qué medida los patrones de especialización de la región se están orientando al aprovechamiento de las oportunidades

${ }^{4}$ Para ahondar en el tema, véase, por ejemplo, Ffrench-Davis (1990). disponibles en los mercados mundiales, así como a examinar los factores que están detrás de las tendencias observadas en esa materia.

El resto del artículo se organiza del siguiente modo. En la sección II se presenta un breve análisis de la evolución de las exportaciones mundiales y, particularmente, latinoamericanas de los SIC en la última década. La sección III, que es el corazón de este trabajo, tiene como objetivo analizar la competitividad de la región en el comercio de los sIC mediante el uso de la metodología TradecAN. En la sección IV se repasa brevemente cuáles son los principales factores determinantes (ex ante) de la competitividad en los sectores de los SIC y se destacan algunos aspectos del posicionamiento de la región. Finalmente, en la sección V se presentan las conclusiones de esta investigación.

\section{II}

\section{El papel de América Latina en el comercio de los SIC}

Antes de examinar brevemente la evolución del comercio mundial y latinoamericano de servicios en los últimos años, es preciso subrayar algunas cuestiones con respecto a las estadísticas disponibles en esa materia. Como es bien sabido, en dichas estadísticas aún no se logra reflejar los volúmenes reales del intercambio mundial de servicios. Esto se debe a un conjunto de factores, tales como: i) muchos de estos servicios son actividades nuevas, y todavía no existe el consenso suficiente acerca de las mejores formas de medirlas; ii) gran parte de estas exportaciones son de naturaleza intangible, lo que dificulta su registro; y iii) las transacciones internacionales de servicios se producen bajo diversas modalidades, no siempre relevadas de forma completa y homogénea en las cifras oficiales (López, Ramos y Torre, 2009). Asimismo, estos problemas pueden verse agudizados debido a que una parte considerable del comercio de servicios es de naturaleza intrafirma, que usualmente se hace a precios de transferencia y a veces ni siquiera es registrada en función de consideraciones impositivas, contables y de otro tipo. Por lo demás, las estadísticas disponibles para comparaciones internacionales operan con un nivel aún excesivo de agregación, lo que hace difícil realizar análisis más finos sobre el comercio de los SIC.

Pese a estas dificultades, de todos modos se cuenta con una cantidad de información suficiente como para proceder al análisis de este estudio y presentar algunas conclusiones respecto del lugar de América Latina en el comercio de los SIC y sus niveles de competitividad ${ }^{5}$.

A partir de la firma del Acuerdo General sobre el Comercio de Servicios (GATs por sus siglas en inglés) a mediados de los años noventa, se reconocen cuatro formas de provisión de servicios a nivel internacional: el modo 1, que abarca al comercio transfronterizo del servicio (muchas veces ligado al intercambio por medio de las TIC); el modo 2, que trata del consumo en el exterior, es decir, cuando el cliente se traslada al país de origen para abastecerse del servicio (por ejemplo, desplazamiento de pacientes en búsqueda de tratamientos médicos); el modo 3, que implica la presencia comercial del proveedor en territorio extranjero, esencialmente por la vía de la inversión extranjera directa (IED), y el modo 4, que registra el movimiento de personas físicas,

\footnotetext{
${ }^{5}$ Hay que tomar en cuenta que los países de la región han avanzado de manera heterogénea en cuanto a la recolección de información sobre el comercio de servicios y presentan estadísticas que difieren significativamente en cuanto a sus niveles de desagregación y cobertura. Ante tal escenario, en este estudio se ha optado por utilizar las estadísticas provistas por la UNCTAD, que permiten un tratamiento homogéneo y la realización de comparaciones entre países, quedando para futuros trabajos la profundización del análisis con datos a nivel nacional para subsectores más específicos dentro de los sic.
} 
es decir, de los proveedores del servicio hacia el país que será su mercado de destino (por ejemplo, el llamado body shopping, la modalidad inicial mediante la cual la industria india de software comenzó a penetrar en los mercados internacionales).

En la práctica, la mayor parte de las transacciones que se registran en las estadísticas de balanza de pagos para la mayoría de las ramas de servicios responde casi exclusivamente al modo 1 , mientras que la cobertura de los modos 2 y 4 suele colapsarse, muchas veces erróneamente, en la categoría de viajes (que incluye sobre todo los ingresos/egresos por turismo). En otras palabras, el segundo y cuarto modo en general no son captados en forma fehaciente por las cifras oficiales, debido a que varias de estas actividades no se registran directamente, o bien están mal registradas a causa de una clasificación inexacta en torno del movimiento de las personas involucradas (como es el caso, por ejemplo, del "turismo médico" o de los servicios educativos que se brindan a extranjeros en el país). Por su parte, la presencia comercial se halla vinculada a los flujos y al acervo de IED $^{6}$. Finalmente, existe una multiplicidad de servicios (ingeniería, diseño, marketing, entre otros) que se comercializan mediante su incorporación en ciertos bienes materiales, quedando así registrados como parte de las ventas de aquellos.

En función de este contexto, el presente estudio se concentra en el comercio basado en el modo 1. Dicho comercio pasó de 1,5 billones de dólares en el año 2000 a 4,4 billones en 2012, de los cuales 1,6 billones corresponden a SIC (según datos recopilados por la UNCTAD). A su vez, mientras que la tasa de crecimiento anual acumulativa del comercio de servicios en el período 2000-2011 fue apenas superior a la de bienes $(9,3 \%$ comparada con 9,1\%), la de los SIC superó ampliamente ambos registros $(11,6 \%)$. Si se observa el entorno de la crisis financiera internacional, se aprecia que los servicios vinculados directamente al intercambio de bienes (el transporte y los servicios financieros) y el turismo tuvieron las mayores caídas, no así los servicios empresariales, profesionales y técnicos que se expandieron incluso en el contexto de la crisis (Borchert y Mattoo, 2010). En la misma línea, datos de consultoras y organismos internacionales sugieren que el comercio en los segmentos de subcontratación de tecnologías de la información (ITO por sus siglas en inglés) y relocalización de procesos de negocios (BPO por sus siglas en inglés), los dos mayores

\footnotetext{
${ }^{6}$ Una manera de aproximarse al comercio de servicios por la vía del modo 3 es utilizar el llamado comercio de filiales extranjeras (FATS por sus siglas en inglés).
}

en cuanto a volumen dentro de los SIC, siguió creciendo en este período de turbulencias internacionales (Gereffi y Fernández-Stark, 2010b; Muthal, 2011).

¿Qué ocurre en el caso de América Latina? La región (incluido el Caribe) tiene una baja participación en el mercado global de los SIC (2,9\% en 2012), aunque levemente mayor a la que tenía en el año 2000 (2,3\%). Esta cifra se compara desfavorablemente con la observada en Asia en desarrollo (27\% en 2012) ${ }^{7}$ (sobre la base de datos de la UnCTAD). Por otra parte, el patrón de exportaciones de servicios de la región tiene aún una base marcadamente asentada en los sectores tradicionales (transporte y viajes), aunque el peso de los SIC ha venido creciendo en la última década y en realidad las mayores diferencias absolutas con el promedio mundial se producen en las áreas de regalías (la región no es exportadora de tecnología) y servicios financieros (véase el cuadro 1), sectores no incluidos en el concepto de SIC aquí empleado. En este sentido, los sectores de sic representaban el 26,6\% de las exportaciones latinoamericanas de servicios en 2010, comparado con el 29,3\% del promedio mundial.

En el cuadro 2 se muestra la clasificación de los principales países exportadores en algunos segmentos de interés para este trabajo (incluidos todos los países latinoamericanos para los que hay datos disponibles). Entre los países en desarrollo, se advierte que la India destaca en la exportación de servicios de computación (es el primer exportador mundial), mientras que China lo hace en otros servicios empresariales (que incluye al BPO, el principal mercado global de deslocalización de servicios (offshoring)). A su vez, China aparece sexta en computación y la India duodécima en servicios empresariales. Dentro de América Latina, el Brasil registra cifras relevantes de exportación en el caso de los servicios empresariales (ocupa el puesto 18 en la clasificación mundial). La Argentina figura en el lugar 24 en computación (liderando la clasificación latinoamericana) y el puesto 34 en servicios empresariales. Más atrás se encuentran Chile, Costa Rica, Colombia y el Uruguay, entre otros (alternando ubicaciones y con importantes diferencias de nivel). En tanto, en el caso de los servicios personales, culturales y recreativos (que incluyen salud, educación, audiovisuales, y otros), resaltan Hungría y Turquía entre los primeros 10 lugares. También en este caso, la Argentina es primera en América Latina (ocupando el puesto 19 de la clasificación mundial).

\footnotetext{
7 Las diferencias relativas son aún mayores que en el caso de las exportaciones industriales, donde América Latina y el Caribe participa con un $4,3 \%$ del mercado mundial, en comparación con el 37,1\% de Asia en desarrollo (sobre la base de datos de la UNCTAD).
} 
CUADRO 1

Exportaciones de servicios a nivel mundial y de América Latina, 2000-2010

(Composición y tasa de crecimiento anual acumulativo (TCCA) en porcentajes)

\begin{tabular}{|c|c|c|c|c|c|c|c|c|}
\hline \multirow{3}{*}{ Categoría } & \multicolumn{4}{|c|}{ Exportaciones mundiales } & \multicolumn{4}{|c|}{ Exportaciones de América Latina } \\
\hline & \multicolumn{3}{|c|}{ Porcentaje del total } & \multirow{2}{*}{$\begin{array}{c}\text { TCAA } \\
(2000-2010)\end{array}$} & \multicolumn{3}{|c|}{ Porcentaje del total } & \multirow{2}{*}{$\begin{array}{c}\text { TCAA } \\
(2000-2010)\end{array}$} \\
\hline & 2000 & 2005 & 2010 & & 2000 & 2005 & 2010 & \\
\hline Transporte & 22,6 & 22,3 & 20,7 & 8,7 & 19,8 & 22,7 & 21,2 & 8,8 \\
\hline Viajes & 31,6 & 27,2 & 24,9 & 7,1 & 47,0 & 46,7 & 40,1 & 6,4 \\
\hline Comunicaciones & 2,1 & 2,2 & 2,4 & 11,1 & 5,4 & 3,5 & 2,9 & 1,6 \\
\hline Servicios financieros y seguros & 7,9 & 8,6 & 9,2 & 11,3 & 6,7 & 4,5 & 5,5 & 6,0 \\
\hline Construcción & 1,9 & 2,1 & 2,3 & 12 & 0,5 & 0,1 & 0,1 & $-6,9$ \\
\hline Computación e informática & 2,8 & 3,3 & 4,4 & 14,7 & 0,6 & 1,2 & 2,8 & 25,5 \\
\hline Regalías y licencias & 6,0 & 6,2 & 6,5 & 10,6 & 0,9 & 0,6 & 0,9 & 7,9 \\
\hline Otros servicios empresariales & 21,0 & 21,9 & 24,1 & 11,2 & 14,6 & 16,3 & 23,0 & 13,1 \\
\hline Servicios personales, culturales, recreativos & 0,9 & 0,8 & 0,7 & 7,3 & 1,0 & 1,2 & 0,7 & 5,2 \\
\hline Servicios de gobierno (+ residuos) & 3,2 & 5,4 & 4,8 & 14,3 & 3,5 & 3,2 & 2,8 & 5,8 \\
\hline
\end{tabular}

Fuente: elaboración propia sobre la base de datos de la Conferencia de las Naciones Unidas sobre Comercio y Desarrollo (UNCTAD).

CUADRO 2

Principales exportadores de SIC, 2010

(En millones de dólares)

\begin{tabular}{|c|c|c|c|c|c|c|c|c|}
\hline \multicolumn{3}{|c|}{ Computación e información } & \multicolumn{3}{|c|}{ Otros servicios empresariales } & \multicolumn{3}{|c|}{ Personales, culturales y recreativos } \\
\hline 1 & India & 56701,2 & 1 & Estados Unidos & 100476,0 & 1 & Estados Unidos & 14563,9 \\
\hline 2 & Irlanda & 37250,8 & 2 & Alemania & 74399,5 & 2 & Reino Unido & 4080,6 \\
\hline 3 & Alemania & 16305,0 & 3 & Reino Unido & 73416,8 & 3 & Canadá & 2198,3 \\
\hline 4 & Estados Unidos & 13830,2 & 4 & China & 61241,6 & 4 & Francia & 1965,4 \\
\hline 5 & Reino Unido & 11518,5 & 5 & Singapur & 43851,3 & 5 & España & 1774,7 \\
\hline 6 & China & 9256,3 & 6 & Japón & 42547,4 & 6 & Malta & 1382,7 \\
\hline 7 & Israel & 7699,5 & 7 & Suiza & 38879,6 & 7 & Luxemburgo & 1352,2 \\
\hline 8 & Suecia & 6660,5 & 8 & Holanda & 32947,8 & 8 & Hungría & 1259,5 \\
\hline 9 & España & 6407,6 & 9 & Francia & 32603,4 & 9 & Alemania & 1074,1 \\
\hline 10 & Holanda & 6155,1 & 10 & Irlanda & 29705,5 & 10 & Turquía & 912,0 \\
\hline 21 & Francia & 1398,6 & 12 & India & 28984,6 & 19 & Argentina & 355,8 \\
\hline 24 & Argentina & 1248,5 & 18 & Brasil & 15776,5 & 21 & India & 334,6 \\
\hline 26 & Costa Rica & 1070,7 & 34 & Argentina & 3910,0 & 32 & China & 122,9 \\
\hline 43 & Brasil & 209,8 & 41 & Chile & 1865,4 & 34 & Brasil & 108,3 \\
\hline 46 & Uruguay & 179,8 & 52 & Costa Rica & 688,5 & 38 & Chile & 86,8 \\
\hline 53 & Chile & 90,7 & 55 & Colombia & 612,1 & 39 & Colombia & 84,2 \\
\hline 57 & Colombia & 45,9 & 58 & Paraguay & 544,2 & 41 & México & 80,0 \\
\hline 66 & Panamá & 24,8 & 66 & Perú & 333,3 & 61 & Paraguay & 14,0 \\
\hline 67 & Perú & 20,9 & 76 & Panamá & 202,6 & 64 & Honduras & 11,7 \\
\hline 69 & Guatemala & 11,7 & 79 & Uruguay & 180,4 & 68 & $\begin{array}{l}\text { Venezuela (República } \\
\text { Bolivariana de) }\end{array}$ & 6,0 \\
\hline \multirow[t]{2}{*}{72} & Venezuela (República & & 81 & Venezuela (República & & 71 & Perú & 3,9 \\
\hline & Bolivariana de) & 9,0 & & Bolivariana de) & 155,0 & & & \\
\hline 77 & Honduras & 4,5 & 90 & Guatemala & 69,6 & 75 & $\begin{array}{l}\text { Bolivia (Estado } \\
\text { Plurinacional de) }\end{array}$ & 1,7 \\
\hline 78 & Paraguay & 4,4 & 100 & El Salvador & 29,6 & 82 & Uruguay & 0,4 \\
\hline \multirow[t]{2}{*}{86} & El Salvador & 0,7 & 106 & Bolivia (Estado & & 84 & Costa Rica & 0,1 \\
\hline & & & & Plurinacional de) & 16,6 & & & \\
\hline \multirow[t]{2}{*}{87} & Bolivia (Estado & & 111 & Honduras & 10,3 & 86 & El Salvador & 0,01 \\
\hline & Plurinacional de) & 0,7 & & & & & & \\
\hline
\end{tabular}

Fuente: elaboración propia sobre la base de datos de la Conferencia de las Naciones Unidas sobre Comercio y Desarrollo (UNCTAD). SIC: servicios intensivos en conocimiento. 


\section{III}

\section{La competitividad de las exportaciones de SIC en América Latina}

Como se señaló anteriormente, el objetivo de este estudio es evaluar la competitividad internacional de América Latina en el sector de los SIC, procurando aportar al debate alguna nueva evidencia sobre el patrón de especialización comercial y la generación de ventajas comparativas (dinámicas) en estas actividades.

Una forma usual para encauzar las comparaciones sectoriales (y, por qué no, las políticas) es colocar el foco en aquellas funciones de servicios que aportan un mayor valor agregado. Aunque ya se han hecho algunas contribuciones al respecto, como, por ejemplo, el ordenamiento sugerido por Gereffi y Fernández-Stark (2010a y 2010b) según la intensidad de valor agregado de diferentes actividades de servicios, la utilización de este diagrama para graficar la situación argentina por López, Niembro y Ramos (2011), o su aplicación dinámica (a cuatro países) en Fernández-Stark, Bamber y Gereffi (2011), lo cierto es que la escasa información disponible y, particularmente, su alto nivel de agregación no permiten pasar de los estudios de casos a comparaciones más generales. En este sentido, las investigaciones mencionadas se basan en trabajos de campo y análisis de literatura y fuentes secundarias para examinar la realidad de cada país o sector.

En tanto, también se han presentado trabajos cuyo propósito consiste en analizar comparativamente la situación de diferentes sectores de SIC en la región (López, Ramos y Torre, 2009; López y Ramos, 2010), y otros en que se procura examinar, a la luz de la evidencia disponible en la literatura teórica y empírica sobre el tema, el posicionamiento de América Latina en las distintas áreas que inciden en la competitividad en estos sectores (López y Ramos, 2013).

Otra forma de analizar el tema es observar lo que señalan las estadísticas de comercio y emplear algunos indicadores usualmente utilizados en la literatura respectiva. El primero es el bien conocido indicador de "ventajas comparativas reveladas" 8 , que pese a sus limitaciones, puede servir como punto de partida para

${ }^{8}$ Aquí se utiliza una variante de este tipo de indicadores que considera las exportaciones, habiendo otros que se basan en la información de saldos comerciales. conocer los patrones de especialización de los principales países exportadores (véase el cuadro 3$)^{9}$.

Previsiblemente, se observa que la India, Irlanda e Israel destacan en el caso de los servicios de computación (las "3 I" según se las conoce en la literatura sobre esta industria), en tanto que en servicios empresariales hay varios países en desarrollo — especialmente de Asiaen los primeros lugares de la clasificación. En servicios personales, culturales y recreativos, el dominio de los países en desarrollo es todavía mayor, incluidas las economías en transición. Dentro de América Latina, Costa Rica, la Argentina y el Uruguay destacan en software, mientras que el Brasil aparece primero en otros servicios empresariales y la Argentina en quinto lugar en servicios personales, culturales y recreativos. Esto indica que varios países latinoamericanos tienen ventajas en los mercados de los SIC, aunque por distintos factores (sobre todo de tamaño) no alcanzan aún a ocupar lugares de privilegio en las clasificaciones por montos exportados.

En suma, si bien los países de América Latina tienen una participación reducida en el comercio mundial de servicios, hay casos exitosos en que se ha logrado consolidar ventajas comparativas y se ha penetrado en mercados dinámicos dentro del mundo de los SIC.

Como ya se mencionó, el indicador de ventajas comparativas reveladas tiene, entre sus limitaciones, la de solo permitir un análisis estático de los niveles de competitividad. Una vía alternativa para avanzar en la superación de esta limitación es diferenciar los patrones comerciales según el dinamismo de los respectivos

${ }^{9}$ El índice se obtiene a partir de la siguiente fórmula:

$$
V C R_{i j}=\frac{X_{i j} / \sum_{i} X_{i j}}{\sum_{j} X_{i j} / \sum_{i} \sum_{j} X_{i j}}
$$

donde $X_{i j}$ representa las exportaciones del sector de servicios $i$ desde el país $j$. El numerador refleja entonces la participación de las exportaciones de un respectivo sector en el agregado de las exportaciones de servicios del país, mientras que el denominador da cuenta de la porción de este sector en las exportaciones mundiales de servicios.

Cuando $V C R_{i j}$ es superior a la unidad, existen ventajas comparativas del país $i$ en el servicio $j$. Si $V C R_{i j}$ está entre 1 y 2 , se dice que el país tiene una ventaja comparativa débil; cuando se encuentra entre 2 y 3, el país tiene una fuerte ventaja comparativa; y si es mayor que 3, la ventaja comparativa es muy marcada. 
Clasificación por "ventajas comparativas reveladas" en los SIC, 2010

\begin{tabular}{|c|c|c|c|c|c|c|c|c|}
\hline \multicolumn{3}{|c|}{ Computación e información } & \multicolumn{3}{|c|}{ Otros servicios empresariales } & \multicolumn{3}{|c|}{ Personales, culturales y recreativos } \\
\hline 1 & India & 7,9 & 1 & Brasil & 2,1 & 1 & Malta & 32,9 \\
\hline 2 & Irlanda & 6,6 & 2 & Filipinas & 2,0 & 2 & Hungría & 6,3 \\
\hline 3 & Israel & 5,4 & 3 & Taiwán & 2,0 & 3 & Serbia & 4,8 \\
\hline 4 & Costa Rica & 4,4 & 4 & Suiza & 1,9 & 4 & Canadá & 3,0 \\
\hline 5 & Finlandia & 4,2 & 5 & Suecia & 1,7 & 5 & Argentina & 2,6 \\
\hline 6 & Filipinas & 2,8 & 6 & Singapur & 1,6 & 6 & Estados Unidos & 2,5 \\
\hline 7 & Sri Lanka & 1,9 & 7 & Paraguay & 1,5 & 7 & Turquía & 2,5 \\
\hline 8 & Rumania & 1,8 & 8 & Bermuda & 1,5 & 8 & Nueva Zelanda & 2,4 \\
\hline 9 & Suecia & 1,8 & 9 & China & 1,5 & 9 & Azerbaiyán & 2,2 \\
\hline 10 & Argentina & 1,6 & 10 & Bélgica & 1,5 & 10 & Luxemburgo & 1,9 \\
\hline 11 & Uruguay & 1,2 & 22 & Argentina & 1,2 & 11 & Colombia & 1,8 \\
\hline 51 & Colombia & 0,2 & 46 & Chile & 0,7 & 21 & Honduras & 1,1 \\
\hline 55 & Chile & 0,1 & 52 & Costa Rica & 0,7 & 25 & Paraguay & 0,9 \\
\hline 59 & Brasil & 0,1 & 55 & Colombia & 0,6 & 30 & Chile & 0,8 \\
\hline \multirow[t]{2}{*}{62} & Perú & 0,1 & 68 & Venezuela (República & & 42 & México & 0,5 \\
\hline & & & & Bolivariana de) & 0,4 & & & \\
\hline \multirow[t]{2}{*}{63} & Guatemala & 0,1 & 69 & Perú & 0,4 & 51 & Venezuela (República & \\
\hline & & & & & & & Bolivariana de) & 0,3 \\
\hline \multirow[t]{2}{*}{64} & Venezuela (República & & 74 & Uruguay & 0,3 & 52 & Brasil & 0,3 \\
\hline & Bolivariana de) & 0,1 & & & & & & \\
\hline 67 & Honduras & 0,1 & & & & 63 & Perú & 0,1 \\
\hline
\end{tabular}

Fuente: elaboración propia sobre la base de datos de la Conferencia de las Naciones Unidas sobre Comercio y Desarrollo (UNCTAD).

SIC: servicios intensivos en conocimiento.

mercados internacionales. Como señala Porta (2005, págs. 12-13), el hecho de introducir en el análisis a la evolución de la "demanda mundial" (dinámica, estancada o en retroceso) permite reconsiderar ciertos aspectos de la especialización productiva y comercial, entendiéndose que algunos posibles problemas de competitividad internacional bien podrían estar reflejando una débil o inadecuada especialización del comercio del país en cuestión. Por su parte, Bianco (2007) destaca que el avance de las economías hacia sectores, funciones o ambos con mercados externos crecientes representa un indicador de ganancias estructurales de competitividad.

Una forma de análisis de la competitividad internacional que se ajusta a los objetivos del presente trabajo (y que, como se mostrará, puede amoldarse a las estadísticas disponibles) es la metodología conocida como Tradecan (Trade Competitive Analysis of Nations), que fue creada en 1990 por la Comisión Económica para América Latina y el Caribe (CEPAL) para analizar, principalmente, la competitividad en el comercio de bienes ${ }^{10}$. A partir de esta metodología, se procura tener una visión dinámica de la competitividad, en la medida en que los países consiguen (o no) acrecentar sus cuotas de mercado en los sectores más (o menos) pujantes en términos de crecimiento de la demanda internacional.

\footnotetext{
10 Vale destacar que en el análisis según la metodología TradecAN no se evalúan las razones por las que los países ganan o pierden posiciones en el comercio internacional.
}

De esta forma, se distinguen cuatro categorías: i) "estrellas nacientes": sectores dinámicos (aumentan su participación en las importaciones mundiales) en los que crece la cuota de mercado del país; ii) "estrellas menguantes": sectores estacionarios o declinantes (se reduce su porcentaje en las importaciones mundiales) en los que se incrementa la cuota de mercado del país; iii) "oportunidades perdidas": sectores dinámicos donde disminuye la participación de mercado, y iv) "retiradas" (o retrocesos): sectores estacionarios o declinantes en los que decae la cuota de mercado del país.

Para llegar a esta categorización se tendrán en cuenta entonces tanto el dinamismo de los distintos sectores como la modificación de la cuota de mercado de cada país entre 2000 y 2009, utilizando la información disponible en la UNCTAD sobre importaciones y exportaciones según los principales segmentos de servicios ${ }^{11}$. Respecto de la primera de las dimensiones de análisis, los sectores declinantes en este período, en función del porcentaje de las importaciones mundiales que representan, son: transporte, viajes, y servicios personales, culturales y recreativos. En cambio, los segmentos de comunicaciones, construcción, seguros, servicios financieros, computación e información, regalías y licencias, y otros servicios empresariales ganan peso en el comercio mundial.

\footnotetext{
${ }^{11}$ Los datos disponibles no dejan de estar sujetos a las debilidades de relevamiento estadístico antes mencionadas (incluida también la falta de reporte de ciertas categorías en algunos países).
} 
Está claro que los datos utilizados para este ejercicio conllevan un alto nivel de agregación, siendo posible que distintas subcategorías dentro de los segmentos principales se comporten de manera diferente. Especialmente, puede ser que algunas sean dinámicas y otras declinantes y que el dinamismo de todos los subsectores no coincida necesariamente con el del agregado. Más adelante se volverá sobre este tema.

Más allá de las salvedades realizadas, en el gráfico 2.A se puede apreciar qué porcentaje de las exportaciones de servicios por parte de países latinoamericanos representó cada una de las categorías del TradecAN en 2009. El grueso de las exportaciones regionales se concentra incluso hoy en sectores estacionarios o declinantes del comercio global de servicios ("estrellas menguantes" y "retiradas"), mientras que la relevancia de las "estrellas nacientes" (sectores dinámicos en los que se gana cuota de mercado) es generalmente reducida, salvo en los casos del Brasil, la Argentina y Costa Rica, donde esta categoría representa por lo menos el $40 \%$ de las exportaciones de servicios. Por otra parte, en varios países de la región una porción considerable de sus exportaciones se origina en "oportunidades perdidas", es decir, en segmentos dinámicos a nivel internacional pero en los que se ha perdido participación de mercado.

En efecto, el patrón descrito para América Latina no se condice con el de otras regiones en desarrollo y emergentes que han sabido aprovechar en mayor grado el crecimiento del comercio mundial de servicios y el fenómeno de la deslocalización de funciones de servicios, logrando insertarse en el entramado de estas cadenas globales de valor. Como se percibe en el gráfico 2.B, los países asiáticos y, en menor medida, de Europa oriental muestran un mayor porcentaje de exportaciones en "estrellas nacientes" y una participación relativamente reducida de "oportunidades perdidas". Algo similar ocurre entre ciertos países desarrollados, como Irlanda, Finlandia, Suecia o Alemania. En cambio, países como los Estados Unidos de América, el Reino Unido de Gran Bretaña e Irlanda del Norte, Francia y el Japón presentan una mayor proporción de "oportunidades perdidas", lo que evidencia que, si bien todavía concentran gran parte de sus exportaciones en segmentos dinámicos del comercio mundial de servicios, durante la década de 2000 han estado relegando cuotas de mercado en estos sectores, de la mano - previsiblemente-de la deslocalización de estas actividades en terceros países. Lo opuesto a este fenómeno, claro está, son las "estrellas nacientes" antes mencionadas entre los países en desarrollo y algunos desarrollados.

Como antes se mencionó, existe la posibilidad de que las distintas subcategorías de servicios presenten trayectorias diversas, que pueden quedar encubiertas o disimuladas por la evolución de las categorías principales recién utilizadas. A modo de análisis parcial de la dinámica de los distintos subsegmentos de servicios, en el cuadro 4 resalta cómo varió su participación en las importaciones totales de servicios en ciertos mercados
A. América Latina

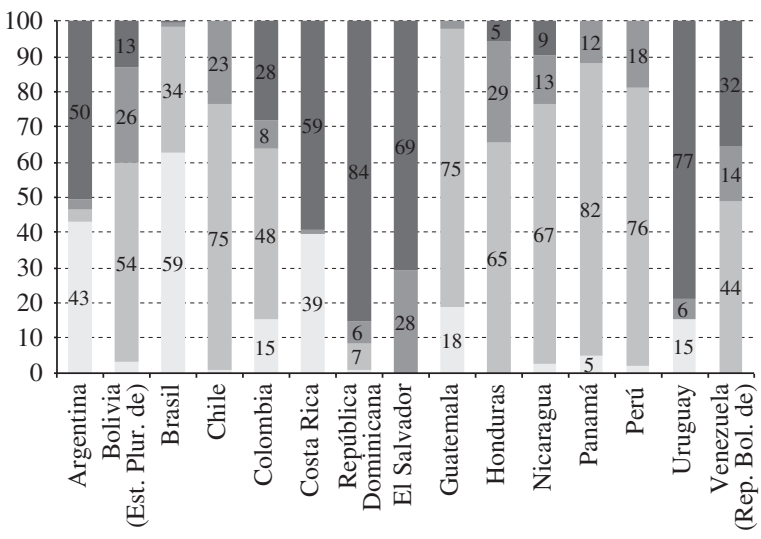

B. Otros países seleccionados

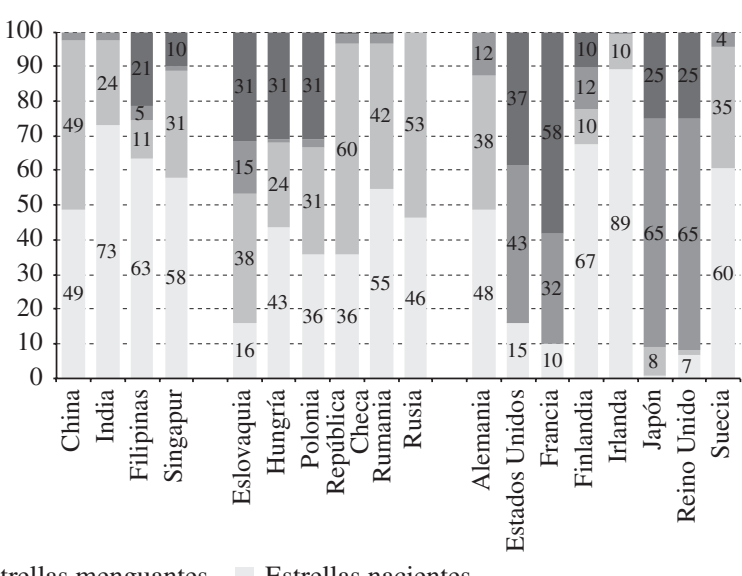

Fuente: elaboración propia sobre la base de datos de la Conferencia de las Naciones Unidas sobre Comercio y Desarrollo (UNCTAD). 
CUADRO 4

Evolución de las importaciones de servicios en algunos mercados seleccionados, según categorías y subcategorías, 2004-2008

(Variación porcentual)

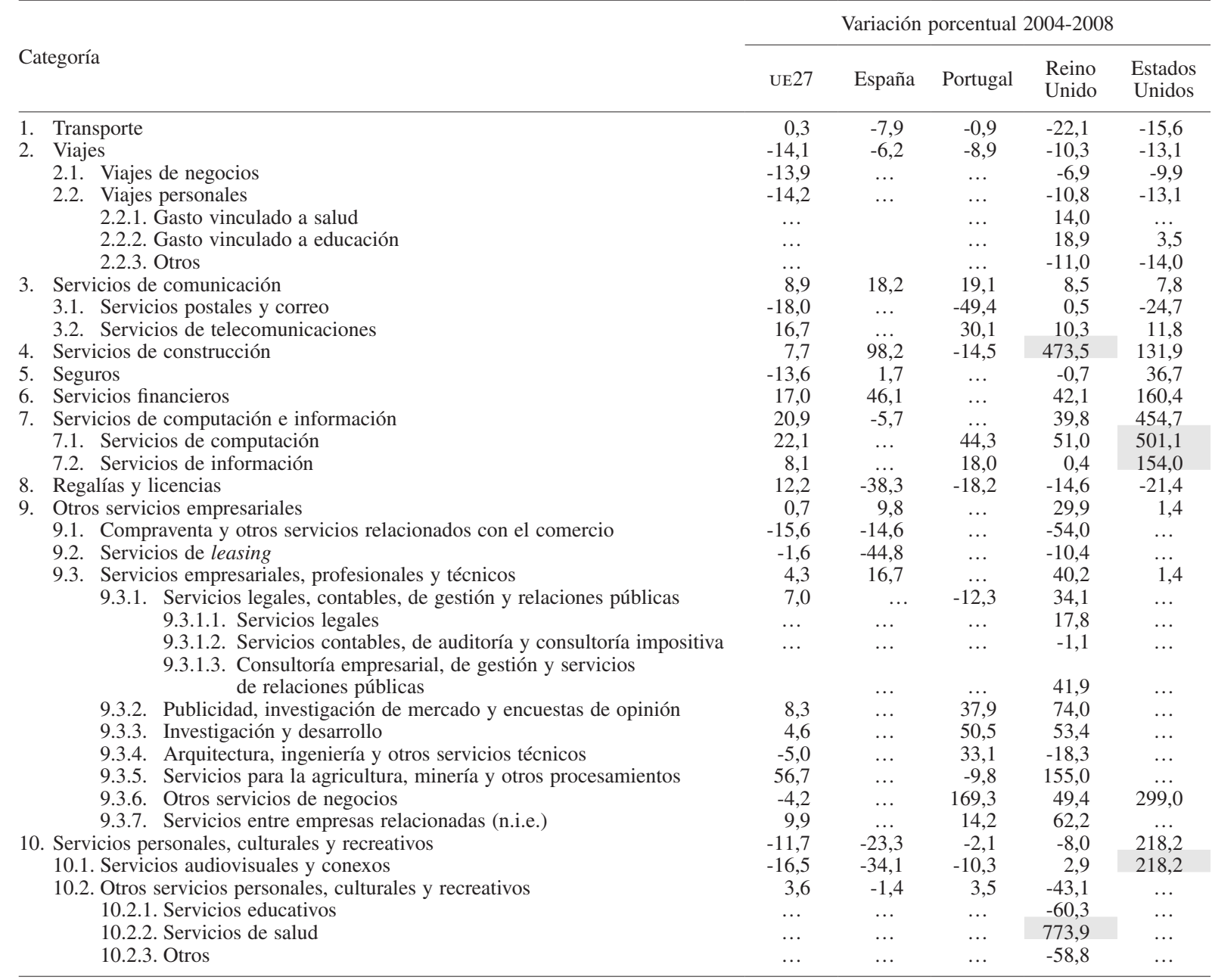

Fuente: elaboración propia sobre la base de datos de United Nations Service Trade.

UE27: 27 economías de la Unión Europea.

n.i.e: datos no incluidos en otra parte.

importadores relevantes ${ }^{12}$, ejercicio que saca a la luz algunas cuestiones interesantes.

Primero, aunque antes en este estudio se consideró a los "servicios personales, culturales y recreativos" como un sector declinante en el agregado global, ahora se advierte que esto no aplica necesariamente a todas

12 En este trabajo se dice "análisis parcial”, pues los datos disponibles no permiten hablar del mercado mundial, como antes se hizo. Otra salvedad es el hecho de que los años de comparación no coinciden, debido a diferencias en la disponibilidad de información según las fuentes. las subcategorías y mercados posibles. En especial, los Estados Unidos de América representan un mercado muy dinámico para las exportaciones de servicios audiovisuales y conexos (y también se ha incrementado el peso de estas importaciones en el Reino Unido). En tanto, la participación de los demás servicios personales, culturales y recreativos ha aumentado entre las importaciones de servicios de la Unión Europea (UE) como un todo (y, en particular, de Portugal).

Segundo, dentro del agregado de "otros servicios empresariales" aparecen importantes diferencias. Por ejemplo, mientras que los servicios de publicidad e 
investigación de mercado e investigación y desarrollo (I+D) muestran un considerable dinamismo, las importaciones del subsegmento de arquitectura, ingeniería y servicios técnicos pierden peso en la UE y el Reino Unido.

Tercero, sobresalen algunos destinos para ciertas exportaciones de servicios: los Estados Unidos de América en el caso de "computación e información" y los ya mencionados servicios audiovisuales; o el Reino Unido para servicios de construcción y de salud (véase qué parte de este último servicio figura, además, como vinculado a "viajes"). Sin embargo, esta lectura de los datos debe tomarse únicamente con fines ilustrativos, puesto que no se dispone de información detallada para muchos destinos/sectores - por ejemplo, en el caso de los Estados Unidos de América, que se sabe es un mercado clave para las exportaciones de servicios de salud (López y otros, 2010).

A continuación, en el gráfico 3 se brinda una óptica alternativa de análisis, que permite matizar algunos resultados del anterior ejercicio de tipo TradecAN no tan favorables para la región. Allí se diferencian ciertos rubros de servicios en particular, distinguiendo a aquellos países que no solo incrementaron su cuota de mercado en las exportaciones mundiales de dichos rubros, sino que también profundizaron la participación de esos segmentos en las exportaciones totales de servicios realizadas por el país en el período 2000-2009.

Como se puede apreciar, a lo largo de la década de 2000 varios países latinoamericanos incrementaron el grado de especialización de sus exportaciones en sectores

GRÁFICO 3

Evolución de la cuota de mercado y participación en exportaciones de SIC de América Latina y países seleccionados, 2000-2009 (Variación porcentual)

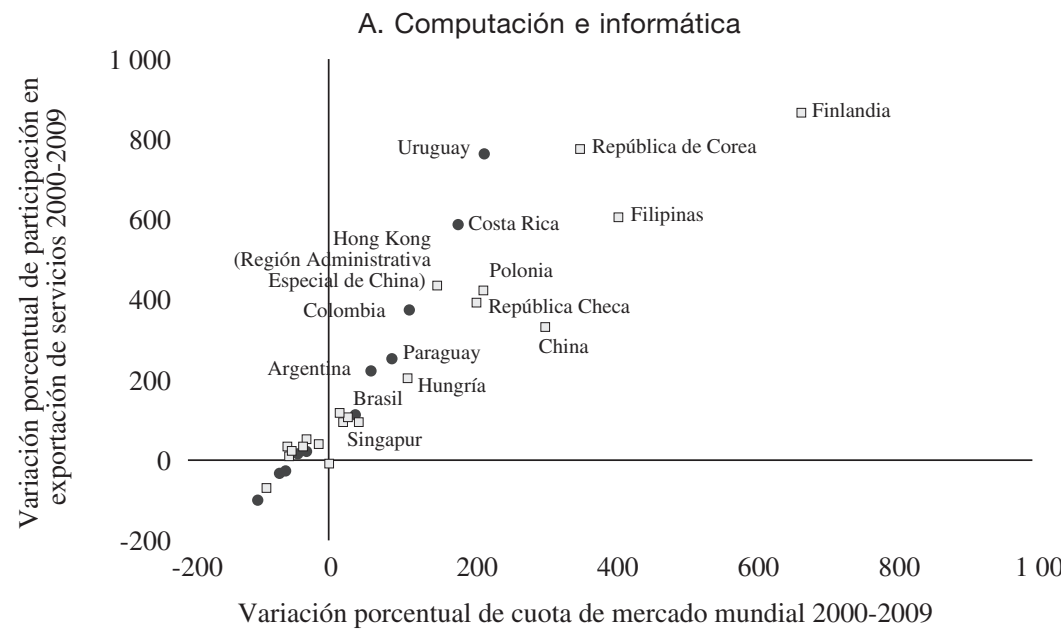

000

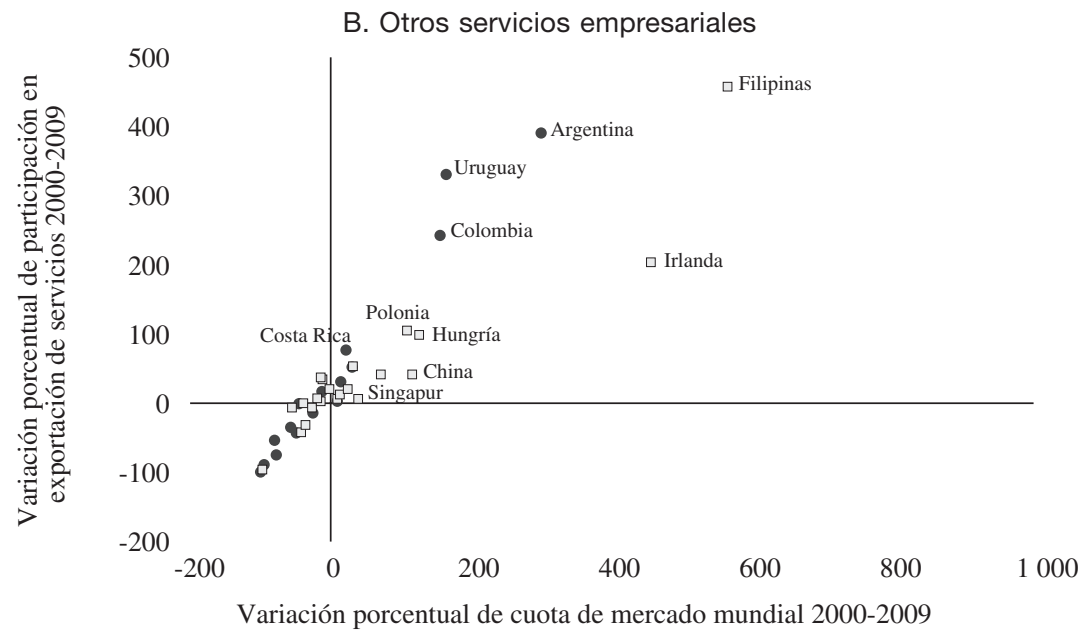




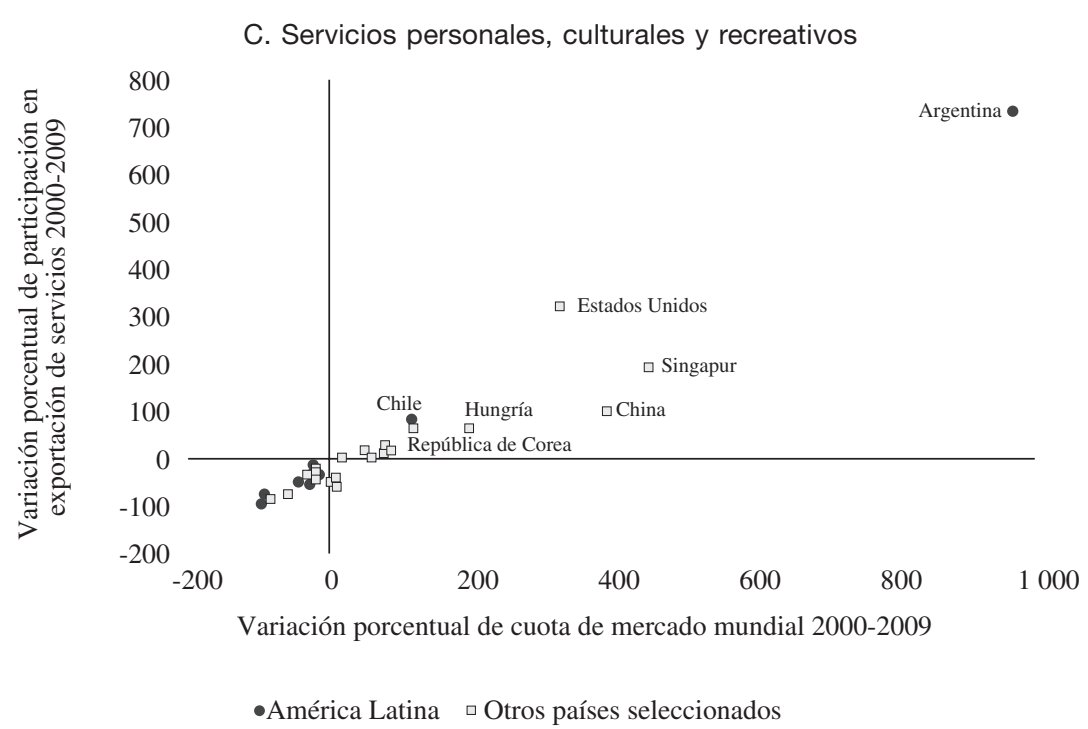

Fuente: elaboración propia sobre la base de datos de la Conferencia de las Naciones Unidas sobre Comercio y Desarrollo (UNCTAD).

SIC: servicios basados en conocimiento.

de los SIC, al tiempo que ganaron mayor participación en el mercado mundial de estos segmentos (véase que, en general, se presenta una relación positiva en la evolución de ambas dimensiones). Incluso, en muchos casos esta trayectoria ocurrió a la par de lo acontecido en otros países (desarrollados y en desarrollo) competidores.

Por último, se hace una breve mención a la dinámica de la competitividad según mercados de destino para las exportaciones latinoamericanas de servicios. Esta se basa en un análisis mediante la lógica de la metodología TradecAN $^{13}$, pero ahora diferenciando por aquellos países-destino más dinámicos a la hora de importar "otros servicios comerciales"14 (es decir, donde entre 2006 y 2008 aumentó la participación de esos rubros en las importaciones totales de servicios), como también por el cambio en la cuota de mercado que ostentan los distintos países latinoamericanos en los países-destino (también en el período 2006-2008).

13 En este sentido, cada uno de los cuadrantes que conforman el gráfico de esta nota puede leerse con la misma lógica que en el análisis TradecAn más convencional, es decir:

\begin{tabular}{|c|c|}
\hline OPORTUNIDADES PERDIDAS & ESTRELLAS NACIENTES \\
\hline RETIRADAS & ESTRELLAS MENGUANTES \\
\hline
\end{tabular}

${ }^{14}$ Dados los "vacíos estadísticos" existentes en cuanto a las categorías reportadas según país de origen de las importaciones, para que el análisis fuera lo más abarcador posible se tuvo que acotar la evaluación a los años 2006-2008 y al grupo de "otros servicios comerciales" (construido como importaciones totales de servicios menos importaciones de los rubros "transporte" y "viajes"), que abarca pero excede a los sIc.
La principal fuente de información para este análisis son las estadísticas de importaciones según su origen, disponibles en United Nations Service Trade y reportadas únicamente por los países de la Organización de Cooperación y Desarrollo Económicos (OCDE), más algunos otros casos puntuales (por ejemplo, Eslovenia y Eslovaquia). En este sentido, una primera limitante es que los gráficos, claro está, no cubren todos los mercados de destino de las exportaciones latinoamericanas, sino especialmente las ventas a países desarrollados y a ciertos emergentes de Europa oriental. No obstante, y si bien es cierto que el comercio de servicios en el interior de la región es un factor importante en el caso de América Latina (a modo de ilustración, véase el trabajo sobre Chile de IDC, 2009), a la hora de expandir el horizonte de las exportaciones latinoamericanas de servicios es preciso apuntar especialmente a los mercados desarrollados, puesto que son los principales importadores mundiales. Es por esto que, a pesar de las limitaciones de cobertura espacial, el gráfico 4 reviste importancia para este trabajo ${ }^{15}$.

\footnotetext{
15 Otro punto a tomar en cuenta es que las declaraciones de origen y destino de las exportaciones de servicios que realizan las empresas están influidas por consideraciones impositivas y de otra índole que pueden en parte dificultar la interpretación de los datos, ya que es posible que una firma declare, por ejemplo, exportaciones a un cierto país, cuando en realidad allí hay un centro de facturación y esos servicios son prestados en la práctica en otro lugar. Sin embargo, para los fines de nuestro análisis entendemos que este tipo de distorsiones no afecta lo central de las conclusiones a las que se arriba.
} 

de otros servicios comerciales (OSC), 2006-2008

(Variación porcentual)

A. Argentina

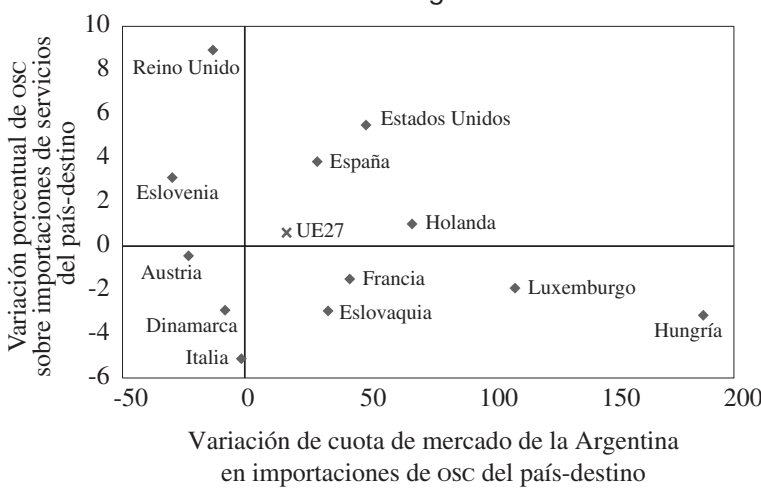

C. México

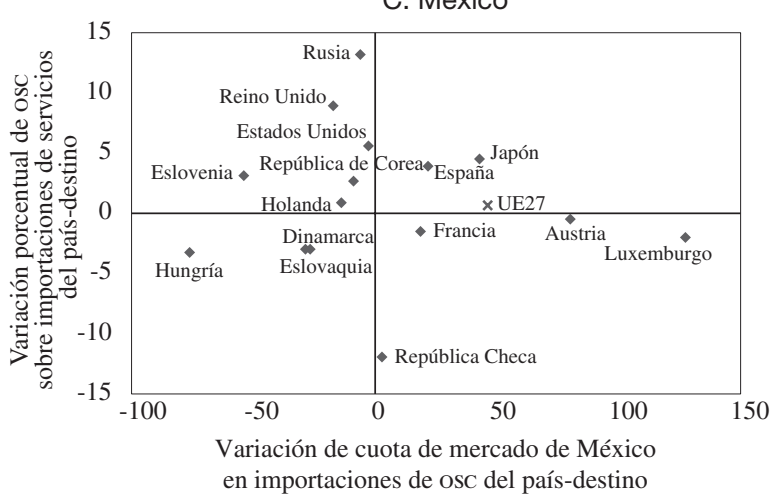

B. Brasil

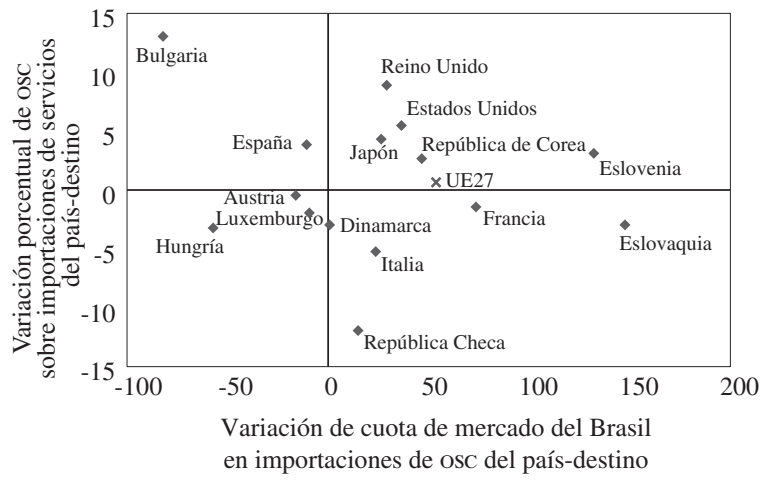

D. Chile

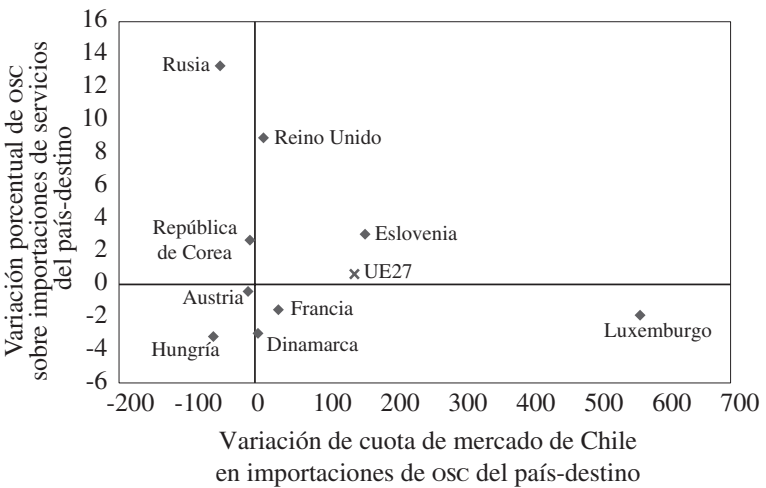

Fuente: elaboración propia sobre la base de datos de United Nations Service Trade y de la Conferencia de las Naciones Unidas sobre Comercio y Desarrollo (UNCTAD).

UE27: 27 economías de la Unión Europea.

Como puede apreciarse, los cuatro países latinoamericanos considerados incrementaron su cuota de participación en el dinámico destino de la UE como un todo (en particular, la Argentina y México mostraron esta misma trayectoria en el caso de España). Respecto de los Estados Unidos de América, este destino aparece como "estrella naciente" para la Argentina y el Brasil, pero se presenta como una “oportunidad (levemente) perdida" para México. En tanto, el Reino Unido es un mercado "estrella naciente" en los casos del Brasil y Chile y "oportunidad perdida" para la Argentina y México, mientras que el Japón figura como "estrella naciente" para el Brasil y México. En definitiva, lo que se observa es que en los últimos años, en un contexto general que aún muestra rezagos competitivos, varios países latinoamericanos han ganado terreno en algunos mercados muy demandantes de importaciones de SIC.

Sin embargo, en trabajos previos se señala que la inserción de la región en los sectores de sic se limita, en general, a segmentos de baja o moderada complejidad tecnológica o que no resultan estratégicos en términos de las actividades globales de las corporaciones que dominan dichas cadenas. Esto es válido para investigación clínica, ingeniería y construcción, programas computacionales o contratación de servicios externos (outsourcing), ya que en todos los casos se replica en mayor o menor medida el mismo patrón (López, Ramos y Torre, 2009; López, Niembro y Ramos, 2011 y 2012). Consecuentemente, ello plantea desafíos adicionales para los países de América Latina desde el punto de vista de la necesidad de "escalar" en las CGV de estos sectores. 


\section{IV}

\section{Los determinantes de la competitividad en los SIC y el posicionamiento de América Latina}

Si se consideran los resultados de los ejercicios presentados en la sección III, es relevante examinar los factores que pueden incidir en el relativamente débil desempeño de la región en los mercados de los SIC, de lo cual pueden surgir lecciones de política útiles a la hora de promover una mayor y mejor inserción de América Latina en estos sectores.

Tanto los reportes producidos por las principales consultoras internacionales como varios trabajos académicos (Doh, Bunyaratavej y Hahn, 2008; Ramasamy y Yeung, 2010; Capik y Drahokoupil, 2011; Liu, Feils y Scholnick, 2011; Kim, Yoon y Lee, 2012) coinciden en la relevancia de los costos (sobre todo laborales) como determinantes de las decisiones de deslocalización de servicios y de la competitividad exportadora. En esta línea, Eichengreen y Gupta (2012) muestran que el tipo de cambio es un factor explicativo clave de las exportaciones de los sic, con mayor fuerza que en el caso de los bienes.

La disponibilidad de capital humano es un segundo factor determinante de las decisiones de deslocalización y de la IED vinculada (Liu, Feils y Scholnick, 2011; Doh, Bunyaratavej y Hahn, 2008), como también de las exportaciones de servicios (Guerrieri y Meliciani, 2005; Nyahoho, 2010; Arora y Bagde, 2011). Goswami y otros (2012) encuentran que el capital humano afecta positivamente a las exportaciones de servicios y que el retorno a la inversión educativa es superior en los países en desarrollo. Es de prever que la relevancia del capital humano aumente con la mayor complejidad de las actividades deslocalizadas (véase Py y Hatem, 2009; Jensen y Pedersen, 2012).

Estudios de caso sobre determinantes de la IED en servicios también reflejan la importancia del dominio del inglés (Ceglowski, 2006; Walsh, 2006; Doh, Bunyaratavej y Hahn, 2008; Capik y Drahokoupil, 2011; Goswami y otros, 2012; Kim, Yoon y Lee, 2012), mientras que en otros trabajos se destaca el peso de la proximidad cultural (Bunyaratavej, Hahn y Doh, 2007; Castellacci, 2010; Nefussi y Schwellnus, 2010). En el caso de la cercanía geográfica (y los husos horarios) no hay una determinación tan clara de la influencia, ya que para ciertas operaciones estar alineado en el mismo huso horario es favorable, mientras que para otras (donde, por ejemplo, importa tener cobertura las 24 horas, los 7 días de la semana) se buscan localizaciones situadas en husos complementarios.

La I+D también es un factor significativo para la competitividad en la exportación de servicios (Popescu y Tachiciu, 2006; Nyahoho, 2010; Seo, Lee y Kim, 2012, al igual que la disponibilidad de infraestructura de TIC apropiada (Guerrieri y Meliciani, 2005; Doh, Bunyaratavej y Hahn, 2008; Ramasamy y Yeung, 2010; Walsh y Yu, 2010).

Por último, el contexto institucional y legal tiene un papel central en las decisiones de localización, ya que dada la naturaleza intangible y poco estandarizada de ciertos servicios, su comercio suele estar asociado a problemas de información asimétrica (Kimura y Lee, 2006; Kolstad y Villanger, 2007; Kandilov y Grennes, 2010; Liu, Feils y Scholnick, 2011). Así, los servicios menos rutinarios y más complejos, que requieren mayor "personalización" o "customización", se deslocalizan preferentemente hacia países con mejor calidad institucional. No obstante, la reducción de los problemas de información asimétrica puede lograrse en parte a través de certificaciones ${ }^{16} \mathrm{o}$ mediante la asociación con redes de prestigio locales, internacionales o ambas (Conti, Turco y Maggioni, 2010).

¿Cómo se posicionan los países de América Latina en los distintos aspectos que contribuyen a la competitividad en estos sectores? Una forma de aproximarse a este tema es mediante las clasificaciones de competitividad que algunas consultoras producen al relevar las localizaciones consideradas más aptas para exportar los sic. En el cuadro 5 se resumen los datos para los países de la región incluidos en el índice elaborado por A.T. Kearney (la más conocida de las clasificaciones), observándose que todos ellos están mal posicionados en nivel educativo y lo mismo ocurre (con la salvedad de Chile) en el entorno de negocios, que incluye temas de propiedad intelectual y exposición cultural. En cambio, el desempeño mejora en materia de capacidad y disponibilidad de la fuerza

\footnotetext{
16 En referencia, por ejemplo, a las certificaciones de calidad CMMI (Capability Maturity Model Integration) para software y servicios informáticos, escM-SP (eSourcing Capabibity Model for Service Providers) para BPO o la acreditación de la Joint Commission International para el turismo médico.
} 
Países latinoamericanos en el Índice A.T. Kearney, 2011

(Posiciones relativas en distintas categorías)

\begin{tabular}{lccccccccc}
\hline & Argentina & Brasil & Chile & Colombia Costa Rica & México & Panamá & Uruguay \\
\hline Índice general & 30 & 12 & 10 & 43 & 19 & 6 & 34 & 41 \\
1. Entorno de negocios & 46 & 30 & 18 & 40 & 25 & 28 & 26 & 29 \\
1.1. Riesgo país & 44 & 28 & 6 & 43 & 22 & 25 & 26 & 32 \\
1.2. Infraestructura & 34 & 33 & 14 & 28 & 29 & 44 & 20 & 27 \\
1.3. Exposición cultural & 45 & 48 & 44 & 40 & 32 & 33 & 39 & 27 \\
1.4. Seguridad de la propiedad intelectual & 48 & 22 & 27 & 29 & 34 & 26 & 38 & 37 \\
2. Capacidad y disponibilidad de fuerza laboral & 15 & 8 & 24 & 26 & 36 & 14 & 49 & 41 \\
2.1. Experiencia relevante & 13 & 9 & 19 & 21 & 34 & 14 & 45 & 38 \\
2.2. Tamaño y disponibilidad de fuerza laboral & 18 & 4 & 28 & 20 & 41 & 8 & 46 & 42 \\
2.3. Educación & 40 & 39 & 29 & 44 & 30 & 34 & 47 & 31 \\
2.4. Idioma & 12 & 31 & 35 & 36 & 16 & 32 & 38 & 17 \\
3. Atractivo financiero & 26 & 37 & 27 & 31 & 14 & 20 & 19 & 28 \\
3.1. Costos laborales & 23 & 34 & 26 & 29 & 17 & 21 & 15 & 30 \\
3.2. Costos de infraestructura & 31 & 50 & 49 & 45 & 12 & 26 & 28 & 37 \\
3.3. Costos de impuestos y regulaciones & 44 & 35 & 6 & 40 & 16 & 25 & 43 & 9 \\
\hline
\end{tabular}

Fuente: elaboración propia sobre la base de A.T. Kearney, Offshoring Opportunities amid Economic Turbulence. The A.T. Kearney Global Services Location Index, 2011, A.T. Kearney, Inc., 2011.

laboral. Con relación al "idioma", solo la Argentina, Costa Rica y el Uruguay ocupan un lugar medianamente destacado. En atractivo financiero (costos) sobresale Costa Rica. En tanto que Chile y el Uruguay compensan sus mayores costos (laborales y de infraestructura) con una menor carga de impuestos y regulaciones. Finalmente, los temas de infraestructura TIC (calidad y costo) son también cuestiones en las que se requieren avances en la mayor parte de los países. Además, todos ellos cuentan con la ventaja de compartir huso horario con los Estados Unidos de América, lo que los posiciona como plataforma costera (nearshore) para las firmas de dicho país.

Las deficiencias más visibles parecen provenir de los sistemas educativos, puesto que hay varias áreas en las que los países latinoamericanos podrían mejorar sus capacidades exportadoras si existiera más personal con las habilidades requeridas ${ }^{17}$. Como se aprecia en el cuadro 6, además de los rezagos en relación con las otras regiones, dentro de América Latina el panorama de la educación universitaria es heterogéneo. Obviamente existen contrastes bien marcados de escala o volumen (México y el Brasil, por una parte, en relación con Costa Rica y el Uruguay en el extremo opuesto), pero también persisten diferencias con respecto al grado de inclinación de los estudiantes por las carreras científicas y técnicas, como también a la efectividad con que terminan sus estudios universitarios. En este sentido, con un número

17 Un análisis en profundidad de la problemática del caso argentino puede encontrarse en López, Niembro y Ramos (2013). de habitantes relativamente similar, Colombia aventaja a la Argentina en materia de graduados en ciencias e ingenierías, mientras que las cifras de Chile tampoco son muy lejanas a las argentinas, a pesar de tener una población considerablemente menor. No obstante, la región muestra en general un bajo porcentaje de graduados en las áreas de ingeniería y ciencias, lo que pone de manifiesto cuán complejo es subsanar los problemas de insuficiencia de personal para algunos segmentos de los SIC.

Además, existe un evidente rezago en materia de calidad de la educación en América Latina, tal como se evidencia en los resultados de los exámenes del Programa Internacional de Evaluación de Estudiantes (PISA por sus siglas en inglés) administrados por la OCDE. En las pruebas tomadas en 2009, que abarcaron 65 países, las 8 naciones de América Latina participantes se ubicaron entre las 20 peores en matemática y en ciencias (en este último caso, con la excepción de Chile).

Finalmente, como es bien conocido, la región tiene complicaciones en cuanto al funcionamiento de sus sistemas de innovación, que se evidencian, por ejemplo, en el pobre desempeño en materia de indicadores tales como gastos en I+D o patentes.

Si bien los problemas de capital humano y las deficiencias en los sistemas de innovación son más amplios y afectan de manera masiva a la estructura económica regional, a la luz de la discusión previa queda claro que son un obstáculo particularmente gravitante para que América Latina progrese en su inserción en las cadenas de SIC y, en especial, avance a los segmentos más complejos dentro de estas CGV. 


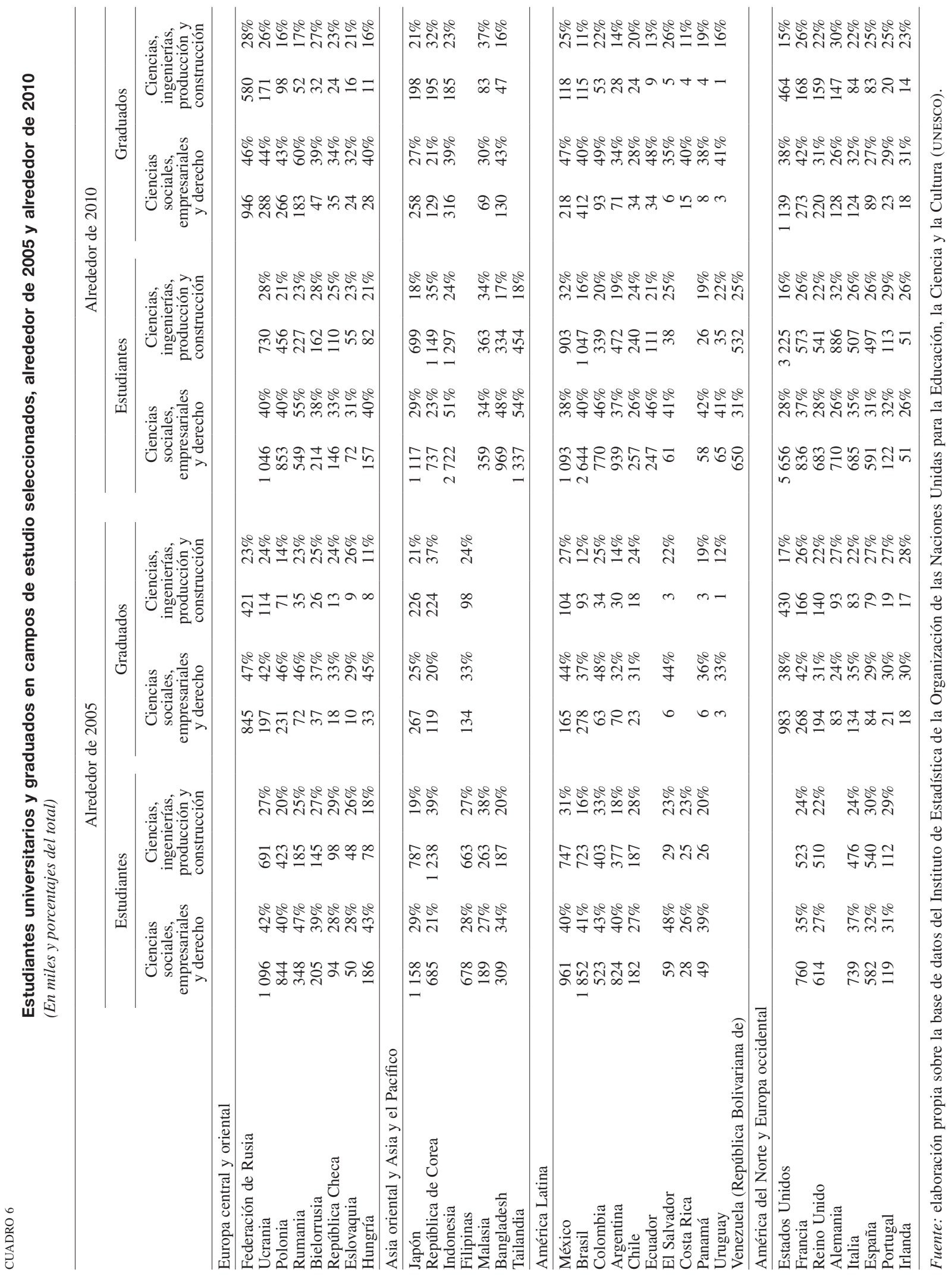




\section{V}

\section{Reflexiones finales}

A lo largo de este trabajo se procuró generar algunas contribuciones para un abordaje dinámico de la competitividad internacional de la región en los SIC, con el objetivo de complementar estudios previos mayormente centrados en el análisis de las "ventajas comparativas (estáticas) reveladas".

En los últimos años, algunos países de América Latina lograron ganar terreno en el comercio mundial de los SIC, incrementando sus cuotas de mercado y penetrando en los principales destinos importadores de estos servicios. Asimismo, el peso de los SIC aumentó, desplazando gradualmente a exportaciones de servicios más tradicionales en toda la región.

Esto ocurre a pesar de que, entre los países latinoamericanos, el peso de los SIC dentro de las ventas totales de servicios suele ser aún reducido en comparación con otros países en desarrollo (como los de Asia o de Europa oriental), tal como surge del relativamente bajo porcentaje de "estrellas nacientes" que se constata en el estudio tipo Tradecan aquí presentado. En parte, esto obedece a un patrón regional de exportaciones de servicios todavía muy dependiente de los sectores más tradicionales (transporte y, especialmente, viajes, segmentos declinantes del comercio mundial). También es probable que -a diferencia de otras naciones que adoptaron con anterioridad la tendencia a la deslocalización de servicios- varios países de América Latina se encuentren atravesando las primeras etapas de aprendizaje e incorporación a estos nuevos mercados, por lo que la profundización de este fenómeno a lo largo de toda la región posiblemente se perciba recién dentro de unos años, siempre y cuando se mantengan algunas condiciones básicas que fundamentan la competitividad en estos sectores. No obstante ello, cabe señalar que la categoría de "servicios empresariales" ocupa un lugar importante entre las "oportunidades perdidas" de algunos países latinoamericanos, hecho que resulta preocupante dado que se trata del mercado más relevante (en términos de volumen) a nivel global.

Con miras al futuro, las perspectivas para la región parecen ser positivas. Todos los pronósticos disponibles coinciden en señalar que los mercados de los SIC van a seguir creciendo a tasas elevadas (véanse, por ejemplo, Muthal, 2011; Volek, 2012). En tanto, América Latina dispone, efectivamente, de una serie de ventajas que la colocan en una buena posición para lograr acrecentar su participación en el comercio internacional de los SIC: i) costos menores que los de los países desarrollados en términos de salarios, inmuebles e infraestructura; ii) una dotación de recursos humanos calificados que, si bien no se compara en cantidad, calidad o en ambas con las de algunos países de Asia o de Europa oriental, es de todos modos suficiente para el desarrollo de un gran número de tareas dentro del ámbito de los SIC, y iii) la proximidad geográfica (incluido el compartir husos horarios) con los Estados Unidos de América y la mayor cercanía cultural con América del Norte y Europa (en comparación con los competidores asiáticos). Estos son atributos generales sobre los cuales la región puede apalancarse para aumentar su nivel de especialización exportadora en los sectores aludidos.

Sin embargo, América Latina - y en particular los países que ya han hecho mayores avances en estos sectores - enfrenta ahora el desafío de mantener y expandir las posiciones adquiridas, sobre la base del desarrollo de ciertos activos que la tornen atractiva para "escalar" en las distintas CGV y comenzar a ganar espacios en los segmentos de mayor sofisticación tecnológica e intensidad de conocimiento, donde la presencia de la región es aún muy limitada.

Ahora bien, avanzar en los eslabones más sofisticados de las cadenas de servicios (escapando gradualmente de las tareas más "commoditizadas", como la mayor parte de las que se engloban en la categoría de relocalización de procesos de negocios) es un desafío complejo. Ello requiere sobre todo progresar en el plano de la cantidad y calidad del capital humano, de modo de consolidar otro tipo de ventajas que vayan más allá de los costos y se basen en activos específicos, talento y capacidades propias para ocupar un lugar diferenciado en estos sectores. Si bien algo de esto ya está sucediendo en algunos casos, se trata de ejemplos dispersos que aún distan de convertirse en una tendencia consolidada, lo que no es sorprendente, ya que se trata de una tarea difícil y que demanda largos tiempos de maduración.

En este sentido, es preciso tener presente que, si la inserción en las CGV y el comercio de servicios se sustentan mayormente sobre la base de los costos laborales, el proceso podría encontrar límites rápidamente si es exitoso. Dicho en otras palabras, en la medida en que la exportación de servicios repercuta favorablemente en el nivel de ingreso per cápita del país exportador (o bien se generen "cuellos de botella" en torno de los recursos 
humanos en los sectores de servicios más pujantes), ello implicará salarios crecientes, que solo si son compensados con similares aumentos de productividad permitirían mantener la competitividad internacional relativa. Pero dado que en buena parte de los SIC se utilizan tecnologías relativamente homogéneas en todo el mundo (básicamente de las TIC y rutinas organizacionales fácilmente transferibles), no pareciera plausible que existan enormes diferencias de productividad entre países que sostengan la competitividad de localizaciones con costos laborales altos o crecientes. Todo esto es más difícil que ocurra, claro está, si la competencia se basa en otros factores diferenciadores y difícilmente replicables por países competidores. La búsqueda de un patrón dinámico y sostenible de exportación de servicios para los países de América Latina debe entonces basarse en el objetivo de potenciar ese tipo de factores y encontrar nichos de mercado apropiados para desarrollarse.

\section{Bibliografía}

A.T. Kearney (2011), Offshoring Opportunities amid Economic Turbulence. The A.T. Kearney Global Services Location Index, 2011, A.T. Kearney, Inc.

Arora, A. y S. Bagde (2011), "Private investment in human capital and industrial development: the case of the Indian software industry", Dimensions of Economic Theory and Policy: Essays for Anjan Mukherji, K. Dastidar, H. Mukhopadhyay y U. Sinha (eds.), Oxford, Oxford University Press.

Baldwin, R. (2011), “Trade and industrialization after globalization's 2nd unbundling: how building and joining a supply chain are different and why it matters", NBER Working Paper, $\mathrm{N}^{\circ}$ 17716, Cambridge, Massachussets, National Bureau of Economic Research.

Bianco C. (2007), “De qué hablamos cuando hablamos de competitividad?", Documento de Trabajo, №31, Buenos Aires, Centro de Estudios sobre Ciencia, Desarrollo y Educación Superior (Centro Redes).

Bobirca, A. y P.G. Miclaus (2007), "A multilevel comparative assessment approach to international services trade competitiveness: the case of Romania and Bulgaria", International Journal of Human and Social Sciences, vol. 1, $\mathrm{N}^{\circ} 1$, World Academy of Science Engineering and Technology.

Borchert, I. y A. Mattoo (2010), "The crisis-resilience of services trade", The Service Industries Journal, vol. 30, No 13 , Taylor $\&$ Francis.

Bunyaratavej, K., E. Hahn y J.P. Doh (2007), "International offshoring of services: a parity study", Journal of International Management, vol. 13, $\mathrm{N}^{\circ} 1$, Amsterdam, Elsevier.

Capik, P. y J. Drahokoupil (2011), "Foreign direct investments in business services: transforming the Visegrád four region into a knowledge-based economy?", European Planning Studies, vol. 19, $\mathrm{N}^{\circ}$ 9, Taylor \& Francis.

Castellacci, F. (2010), "The internationalization of firms in the service industries: Channels, determinants and sectoral patterns", Technological Forecasting and Social Change, vol. 77, $\mathrm{N}^{\circ} 3$, Amsterdam, Elsevier.

Ceglowski, J. (2006), "Does gravity matter in a service economy?", Review of World Economics, vol. 142, $\mathrm{N}^{\circ} 2$, Springer.

Chudnovsky, D. y F. Porta (1990), "La competitividad internacional. Principales cuestiones conceptuales y metodológicas", Documento de Trabajo, $\mathrm{N}^{\circ} 3$, Buenos Aires, Centro de Investigaciones para la Transformación (CENIT).

Conti, G., A.L. Turco y D. Maggioni (2010), "Exporters in services: new evidence from Italian firms", Applied Economics Quarterly, vol. 56, $\mathrm{N}^{\circ} 1$, Berlín, Duncker \& Humblot.

Díaz de la Guardia, C., J. Molero y P. Valadez (2005), "International competitiveness in services in some European countries: basic facts and a preliminary attempt of interpretation", Working Paper, $\mathrm{N}^{\circ}$ 01/05, Madrid, Instituto Complutense de Estudios Internacionales, Universidad Complutense de Madrid.

Doh, J.P., K. Bunyaratavej y E.D. Hahn (2008), "Separable but not equal: the location determinants of discrete services offshoring activities", Journal of International Business Studies, vol. 40, $N^{\circ}$ 6, Palgrave Macmillan.

Eichengreen, B. y P. Gupta (2012), "The real exchange rate and export growth: are services different?", MPRA Paper, $\mathrm{N}^{\circ} 43358$, Munich, University Library of Munich.

Escaith, H. (2008), "Measuring trade in value added in the new industrial economy: statistical implications", MPRA Paper, $\mathrm{N}^{\circ} 14454$, Munich, University Library of Munich.

Fernández-Stark, K., P. Bamber y G. Gereffi (2011), The Offshore Services Global Value Chain: Economic Upgrading and Workforce Development, Durham, Center on Globalization, Governance \& Competitiveness, Universidad de Duke.

Ferraz, J.C., D. Kupfer y L. Haguenauer (1996), Made in Brazil: Desafios competitivos para a indústria, Río de Janeiro, Editora Campus.

Ffrench-Davis, R. (1990), "Ventajas comparativas dinámicas: Un planteamiento neoestructuralista", Cuadernos de la CEPAL, $\mathrm{N}^{\circ}$ 63, Santiago de Chile, Comisión Económica para América Latina y el Caribe (CEPAL).

Gereffi, G. y K. Fernández-Stark (2010a), The Offshore Services Global Value Chain, Durham, Center on Globalization, Governance \& Competitiveness, Universidad de Duke.

(2010b), "The offshore services value chain: developing countries and the crisis", Policy Research Working Paper, $\mathrm{N}^{\circ}$ 5262, Washington, D.C., Banco Mundial.

Goswami, A.G. y otros (2012), "Service exports: are the drivers different for developing countries?", Exporting Services: A Developing Country Perspective, A.G. Goswami, A. Mattoo y S. Saez (eds.), Washington, D.C., Banco Mundial.

Guerrieri, P. y V. Meliciani (2009), "Internationalization, Technology and Business Services Specialization in Europe", Roma.

(2005), "Technology and international competitiveness: the interdependence between manufacturing and producer services", Structural Change and Economic Dynamics, vol. 16, $\mathrm{N}^{\circ} 4$, Amsterdam, Elsevier.

IDC (2009), "La industria de servicios globales en Chile", Santiago de Chile, Corporación de Fomento de la Producción (CORFO).

Jensen, P. y T. Pedersen (2012), "Offshoring and international competitiveness: antecedents of offshoring advanced tasks", Journal of the Academy of Marketing Science, vol. 40, $\mathrm{N}^{\circ} 2$, Springer.

Jiang, W. (2011), "Comparative Analysis on the Competitiveness of Service Trade in China and Japan and Its Enlightenments for China”, 2011 International Conference on Management and Service Science (MASS).

Kandilov, I.T. y T. Grennes (2010), "The determinants of service exports from Central and Eastern Europe", Economics of Transition, vol. 18, $\mathrm{N}^{\circ} 4$, Wiley.

Kim, Y.-D., S. Yoon y K.S. Lee (2012), "International competitiveness of Korea service industry", American Journal of Applied Sciences, vol. $9, \mathrm{~N}^{\circ} 3$, Science Publications. 
Kimura, F. y H.H. Lee (2006), "The gravity equation in international trade in services", Review of World Economics, vol. 142, $\mathrm{N}^{\circ} 1$, Springer.

Kolstad, E. e I. Villanger (2007), "Determinants of foreign direct investment in services", European Journal of Political Economy, vol. 24, $\mathrm{N}^{\circ}$ 2, Amsterdam, Elsevier.

Langhammer, R.J. (2004), "Revealed comparative advantages in the services trade of the United States, the European Union and Japan: what do they tell us?", The Journal of World Investment \& Trade, vol. $5, \mathrm{~N}^{\circ} 6$.

Liu, R., D. Feils y B. Scholnick (2011), "Why are different services outsourced to different countries?", Journal of International Business Studies, vol. 42, Palgrave Macmillan.

López, A. y D. Ramos (2013), “¿Pueden los servicios intensivos en conocimiento ser un nuevo motor de crecimiento en América Latina?", Revista Iberoamericana de Ciencia, Tecnología y Sociedad, vol. 8, $\mathrm{N}^{\circ}$ 24, Buenos Aires, Centro de Estudios sobre Ciencia, Desarrollo y Educación Superior.

(coords.) (2010), La exportación de servicios en América Latina: Los casos de Argentina, Brasil y México, Montevideo, Red Mercosur de Investigaciones Económicas.

López, A., A. Niembro y D. Ramos (2013), "Cadenas globales de valor, offshoring de servicios y rol de los recursos humanos: Lecciones de la Argentina", El sistema argentino de innovación: Instituciones, empresas y redes. El desafío de la creación y apropiación de conocimiento, D. Suárez (ed.), Los Polvorines, Buenos Aires, Ediciones UNGS.

(2012), "Posibilidades de inserción de los países de América Latina en cadenas globales de valor en servicios: Un análisis de las políticas de promoción de exportaciones y atracción de inversiones", Santiago de Chile, Comisión Económica para América Latina y el Caribe (CEPAL).

(2011), "Cadenas globales de valor en el sector servicios: estrategias empresarias e inserción de los países de América Latina”, Integración \& Comercio, $\mathrm{N}^{\circ} 32$, Washington, D.C., Banco Interamericano de Desarrollo (BID), enero-junio.

López, A. y otros (2010), "El comercio mundial de servicios de salud", La exportación de servicios en América Latina: Los casos de Argentina, Brasil y México, A. López y D. Ramos (coords.), Montevideo, Red Mercosur de Investigaciones Económicas.

López, A., D. Ramos e I. Torre (2009), "Las exportaciones de servicios de América Latina y su integración en las cadenas globales de valor", Documento de Proyecto, N 37 (LC/W.240), Santiago de Chile, Comisión Económica para América Latina y el Caribe (CEPAL).
Muthal, H. (2011), "Realizing Opportunities. The Global IT-BPO Sector and the Potential of Latin America \& the Caribbean", Tholons Inc.

Nefussi, B. y C. Schwellnus (2010), "Does FDI in manufacturing cause FDI in business services? Evidence from French firmlevel data", Canadian Journal of Economics, vol. 43, $\mathrm{N}^{\circ} 1$, Quebec, Canadian Economics Association.

Nyahoho, E. (2010), "Determinants of comparative advantage in the international trade of services: an empirical study of the Hecksher-Ohlin approach", Global Economy Journal, vol. $10, \mathrm{~N}^{\circ} 1$.

Popescu, N. y L. Tachiciu (2006), "Research and development as a determinant of services sector competitiveness", Journal for Economic Forecasting, vol. 3, $\mathrm{N}^{\circ} 4$.

Porta, F. (2005), "Especialización productiva e inserción internacional. Evidencias y reflexiones sobre el caso argentino", proyecto FO/ARG/05/012, Programa de las Naciones Unidas para el Desarrollo (PNUD).

Py, L. y F. Hatem (2009), "Where do multinationals locate service and manufacturing activities in Europe and why?", Économie et statistique, $\mathrm{N}^{\circ} 426$.

Ramasamy, B. y M. Yeung (2010), "The determinants of foreign direct investment in services", World Economy, vol. 33, $\mathrm{N}^{\circ} 4$, Wiley Blackwell.

Seo, H.-J., Y.S. Lee y H.S. Kim (2012), "The determinants of export market performance in Organisation for Economic Cooperation and Development service industries", The Service Industries Journal, vol. 32, $\mathrm{N}^{\circ}$ 8, Taylor \& Francis.

Seyoum, B. (2007), "Revealed comparative advantage and competitiveness in services. a study with special emphasis on developing countries", Journal of Economic Studies, vol. 34, $\mathrm{N}^{\circ}$ 5, Emerald.

Volek, T. (2012), "Global Off Shoring and Outsourcing Market Dynamics", Conferencia SOFTECON 2012, Bratislava, 15 de marzo.

Walsh, K. (2006), "Trade in services: does gravity hold? A gravity model approach to estimating barriers to services trade", Discussion Paper Series, $\mathrm{N}^{\circ}$ 183, Institute for International Integration Studies.

Walsh, J.P. y J. Yu (2010), "Determinants of foreign direct investment: a sectoral and institutional approach", IMF Working Paper, $\mathrm{N}^{\circ} 10 / 187$, Washington, D.C., Fondo Monetario Internacional.

Zhang, B., N. Wei y Y. Miao (2010), "China's Service Trade Competitiveness and its Development Strategies", documento presentado en la 2010 WASE International Conference on Information Engineering. 